\title{
ЭФФЕКТИВНОСТЬ ФОНДОВ ПРЯМЫХ ИНВЕСТИЦИЙ НА РАЗВИВАЮЩИХСЯ РЫНКАХ
}

Родионов И.И. ${ }^{1}$, Гусамов С.А. ${ }^{2}$

Из-за отсутствия данных о денежных потоках оценить эффективность ФПИ для развивающихся рынков капитала представляется более сложной задачей, чем для развитых, так как использование традиционных показателей доходности TVPI u IRR невозможно.

В данном исследовании был предложен подход, предполагающий применение скорректированных показателей доходности для ФПИ, который может быть реализован без использования данных о денежных потоках и чистых активов фондов. Предложенные показатели могут быть рассчитаны на основе имеющихся в публичном доступе данных о портфельных сделках фондов.

В исследовании была представлена методология анализа эффективности ФПИ на основе портфельных сделок, а также проведен анализ эмпирических данных на выборке из 1957 сделок стран БРИКС в период с 2000 по 2012 г.

Результаты эмпирического анализа в целом подтверждают ряд фундаментальных характеристик ФПИ, ранее выявленных для развитых рынков капитала, таких как:

- $\quad$ Сделки ФПИ в развивающихся странах являются более рисковыми активами, чем традиционные инструменты.

- Доходность у большинства сделок ниже доходности фондового рынка, однако наиболее успешные существенно опережают рынок.

- Инвестиции фондов выкупа имеют коэффициент $\beta$ меньше единицы, что говорит о малой подверженности системному риску.

- Некоторые характеристики нашли подтверждение только частично:

- $\quad$ Инвестиции венчурных фондов имеют коэффициент $\beta$ больше единицы для рынков Бразилии и Индии, и меньше единицы для России и Китая.

- Доходность инвестиций ВО фондов выше доходности VC фондов в России и Китае. В Индии и Бразилии - результат обратный.

Оставшаяся часть характеристик принципиально отличается от выявленных на развитых рынках капитала:

- Период владения инвестициями для ФПИ развивающихся стран меньше, чем для развитых стран, и в среднем составляет 3,3 года.

Ключевые слова: фонды прямых инвестиций, венчурные фонды, эффективность, оценка активов.

JEL: G12, G23

В последнее время возросло число исследований привлекательности активов, относящихся к альтернативным инвестициям. К ним относятся в том числе вложения в хедж-фонды, фонды недвижимости, товарно-сырьевые активы и их производные, а также вложения в фонды прямых инвестиций (private equity funds). Особенность данного вида активов заключается в том, что они менее выражено коррелируют с традиционными финансовыми активами (акциями и облигациями публичных компаний), поэтому их использование позволяет достичь большей диверсификации портфеля и снизить дисперсию его доходности.

Тема оценки эффективности фондов прямых инвестиций в развитых странах стала активно развиваться в 90-х годах прошлого века, когда появился достаточный набор данных для анализа. В связи с возросшим интересом к альтернативным инвестициям увеличилась активность исследования фондов прямых инвестиций (ФПИ). Изучаются проблемы эффективности (performance) данного инвестиционного инструмента, его доходности, риска, кор-

1. Д-р эконом. наук, профессор кафедры «Экономики и финансов фирмы» НИУ ВШЭ.

2. Аспирант НИУ ВШЭ. 
реляции с традиционными активами, влияния различных факторов на его эффективность. Однако основная масса этих исследований посвящена фондам прямых инвестиций развитых стран, работающим на развитых рынках, в то время как развивающиеся рынки, на наш взгляд, остаются менее изученными.

Малое число работ, а также недостаточная глубина анализа в исследованиях эффективности работы фондов прямых инвестиций в развивающихся странах обусловлены тем, что отсутствует публичная информация о денежных потоках и стоимости чистых активов таких фондов ФПИ развивающихся стран. По этой причине невозможно использовать имеющиеся методы исследования ФПИ развитых стран, основанные на использовании данных о денежных потоках и стоимости чистых активов фондов.

Из-за недостатка академических исследований, а также применимых на практике методик оценки эффективности ФПИ в странах с развивающейся экономикой существует ряд проблем, с которыми сталкиваются инвесторы, рассматривающие возможность размещения своих средств в фонды прямых инвестиций развивающихся стран:

1. дополнительные издержки, связанные с поиском и оценкой деятельности управляющих компаний ФПИ;

2. проблема при выборе управляющих компаний, специализации фонда, времени и географии инвестирования;

3. трудности в оценке доходности, рисков, корреляции с другими активами и других показателей эффективности инвестиций в ФПИ развивающихся стран.

Данные проблемы увеличивают неопределенность, затрудняют расчеты и прогнозирование результатов, таким образом усиливая риски инвестирования в ФПИ развивающихся стран и повышая вероятность отказа инвесторов от участия в привлекательных инвестиционных проектах в этих странах.

Важность снижения неопределенности инвестирования в ФПИ развивающихся стран, проведения более точной оценки рисков, доходности и других показателей эффективности, для повышения привлекательности данного финансового инструмента среди инвесторов делают исследования в данной области актуальными. Академический аспект актуальности исследования заключается в необходимости разработки такого подхода к оценке эффективности ФПИ, который, с одной стороны, будет применим в условиях недостатка данных о ФПИ на развивающихся рынках, а с другой - сможет обеспечить сопоставимость полученных результатов с аналогичными исследованиями для ФПИ развитых стран.

Предлагаемый авторами подход к оценке эффективности ФПИ основывается на анализе произведенных фондами сделок, что позволяет уйти от проблемы недостатка данных о денежных потоках фондов. Для этого предлагается применять два скорректированных показателя доходности: инвестиционный мультипликатор для сделок и среднегодовой рост стоимости актива. Представляется, что данные показатели с высокой степенью достоверности могут служить для сопоставления с показателями TVPI и IRR, которые широко используются в исследованиях эффективности ФПИ на развитых рынках капитала. На основе предложенных показателей возможен анализ распределения доходности фондов, корреляции с доходностью публичных активов, а также продолжительности горизонта инвестирования.

\section{Обзор литературы и уточнение задач исследования}

Под эффективностью ФПИ обычно понимается набор параметров, которыми характеризуются денежные потоки инвестора, связанные с участием в фонде прямых инвестиций в период с начала до конца существования фонда [Gompers and Lerner, 2001]. К таким параметрам относятся:

1. Доходность (возможно использование нескольких показателей для измерения доходности). 
2. Дисперсия и другие характеристики распределения доходности.

3. Корреляция доходности с рыночным портфелем (ковариация, бета).

4. Инвестиционный горизонт (период, в течение которого инвестиции в ФПИ не могут быть конвертированы в денежные средства или другие активы).

Эффективность ФПИ как класса активов (performance of private equity funds as an asset class) определяется исследователями как набор характеристик распределения параметров эффективности для репрезентативной выборки фондов на продолжительном временном интервале.

В исследованиях ФПИ развитых стран применяется традиционный подход к оценке эффективности - подход на уровне фонда. Данный подход основан на изучении денежных потоков, которые поступают в фонд от инвесторов, и потоков, которые распределяются между инвесторами при реализации активов фонда. Данный подход удобен, так как позволяет напрямую работать с денежными потоками ограниченного партнера. Однако важным условием для успешной реализации такого подхода является наличие данных о денежных потоках фонда от момента создания до момента его ликвидации.

Ключевым параметром эффективности актива является его доходность. Для оценки доходности в исследованиях ФПИ на развитых рынках используются несколько основных подходов, из них наиболее часто встречающиеся - это расчет денежных мультипликаторов и оценка внутренней ставки доходности и ее модификаций.

Денежные мультипликаторы показывают приращение первоначального объема инвестиций без учета временного фактора. Наиболее часто используемым мультипликатором является TVPI (total value to paid in capital), который равен отношению суммы всех фактических и потенциальных денежных поступлений к общему объему проинвестированного капитала [Diller and Kaserer, 2008; Kaplan and Schoar 2005; Gompers and Lerner 1999]:

$$
\text { TVPI }=\frac{\sum \text { Distributions }}{\sum \text { Contributions }} \text {. }
$$

Капитал инвестируется для целей приобретения фондом портфельных компаний, а поступления формируются главным образом за счет продажи долей компании путем IPO или стратегическому инвестору. Точный расчет данного показателя требует полной реализации фондом своих активов и погашения всех обязательств перед инвесторами. Однако при промежуточной оценке результатов часто применяются мультипликаторы DPI (Distributions to paid in capital) и RPI (Residual value to paid in capital), где DPI отражает отношение фактических поступлений к проивнестированому капиталу, а RPI - отношение оставшихся чистых активов к проинвестированому капиталу:

$T V P I^{\prime}=D P I+R P I=\frac{\sum \text { Distributions }}{\sum \text { Contributions }}+\frac{\text { Residual Value }}{\sum \text { Contributions }}$.

Показатель TVPI не является объективным измерителем доходности, так как не учитывает временной фактор. Однако его часто используют как вспомогательный индикатор из-за его простоты.

Внутренняя ставка доходности является более удобным показателем, так как учитывает временную структуру денежных потоков [Ellis and Steer, 2011]. Аналогичным образом IRR может быть рассчитана как на основе фактических, так и на основе ожидаемых денежных потоков. Однако внутренняя норма доходности не является безупречным индикатором доходности для инвестиций в ФПИ потому, что предпосылка о реинвестировании положительных денежных потоков под ставку, которая совпадает с IRR, в подавляющем большинстве случаев не соответствует действительности [Ellis and Steer, 2011].

Индекс рентабельности и модифицированная внутренняя ставка доходности частично решают данную проблему, так как при расчете используется внешняя ставка дисконтирования 
- прокси для реальной доходности денежных потоков инвестора вне рассматриваемого проекта, которая приводит все отрицательные потоки к нулевому периоду, а все положительные - к последнему [Phalippou and Zollo, 2005]:

$$
\begin{aligned}
& P I=\frac{\sum_{i}^{N}\left(C F_{i}^{+} \times(1+r)^{N-i}\right)}{\sum_{i}^{N} \frac{C F_{i}^{-}}{(1+r)^{N-i}},} \\
& M I R R=\left(\frac{\sum_{i}^{N}\left(C F_{i}^{+} \times(1+r)^{N-i}\right)}{\sum_{i}^{N} \frac{C F_{i}^{-}}{(1+r)^{N-i}}}\right)^{\frac{1}{N}} .
\end{aligned}
$$

Однако существует проблема корректности определения данной ставки доходности. С одной стороны, для того, чтобы сопоставлять эффективность вложений инвесторов в различные фонды, нужна единая ставка дисконтирования, а с другой стороны, в реальной жизни она определяется возможностями каждого конкретного инвестора.

Исследования распределения показателей доходности и инвестиционного горизонта ФПИ США, Великобритании и Европы позволили определить несколько важных свойств для данного вида активов:

1. Фонды прямых инвестиций могут быть отнесены к альтернативным инвестициям, так как доходность ФПИ слабо коррелирует с традиционными финансовыми активами (акциями и облигации публичных компаний). Это подтверждается исследованиями Джонс и РходесКропф [Jones and Rhodes-Kropf, 2003] на выборке из 1245 фондов CША (VC/BO - 70\%/30\%), Каплан и Шоа [Kaplanand Schoar, 2005] с выборкой из 746 фондов в период с 1980 по 2001 г. (VC/BO - 78\%/22\%), а также рядом других исследований [Gompers and Metrick, 2001; Metrick and Yasuda, 2010].

2. Инвестиции в ФПИ являются более рисковыми инвестициями по сравнению с традиционными финансовыми инструментами, об этом говорят результаты значительного количества исследований, например, Люнгквист и Ричардсон [Ljungqvist and Richardson, 2003], которые проанализировали выборку фондов по данным одного из крупнейших институциональных инвесторов США. При этом Шмидт [Schmidt, 2006] в своей работе делает вывод, что риск ФПИ в основном носит несистематический характер, то есть его можно снизить путем диверсификации.

3. Каплан с соавторами [Kaplan et al., 2002] на выборке в 1240 фондах в США и Европе показали, что распределение показателей доходности среди фондов прямых инвестиций развитых стран неравномерное, имеет «тяжелые хвосты» и смещено вправо, что дает сигнал инвесторам о том, что, несмотря на низкую среднюю доходность по сегменту, существует ряд фондов, чьи показатели доходности значительно превышают средний уровень. К аналогичным выводам пришли, другие исследователи [Richardson, 2003; Jenkinson et al., 2013; Kaplan, and Schoar, 2005], а также ряд других исследователей/

4. Инвестиции в ФПИ неликвидны. Инвестиционный горизонт вложений в ФПИ составляет в среднем 4,5-6 лет. Вторичный рынок по продаже долей участия в ФПИ практически отсутствует [Hwang et al., 2005; Chen et al., 2002].

Научное сообщество не сошлось во мнении, являются ли фонды прямых инвестиций более доходным активом, чем рыночные портфели крупных фондовых бирж. Существует множество работ как в пользу превышения доходности ФПИ над рыночным портфелем [Cochrane, 2005; Hwang et al., 2005; Gro and Gottschalg, 2008; Chen et al., 2002; Kaplan and Schoar, 2005], так и против [Jones and Rhodes-Kropf, 2003; Phalippou and Gottschalg, 2009; Driessen et al., 2008]. 


\section{Гипотезы и анализ эмпирических данных}

Гипотезы сформулированы на основе инвестиционного профиля ФПИ как класса активов, который был составлен на результатах исследований распределения доходности и горизонта инвестирования для ФПИ развитых стран. Таким образом, делается попытка установить соответствие между характеристиками эффективности ФПИ развитых и развивающихся стран.

С позиции инвестора ФПИ как класс инвестиций должен демонстрировать риск и доходность выше рыночной, имея при этом низкую подверженность системному риску. Проведенные исследования в развитых странах частично нашли подтверждение данному факту: риск ФПИ в среднем выше рыночного, но доходность выше рынка демонстрирует только небольшая часть фондов, при этом наиболее успешные фонды существенно опережают рынок. Фонды выкупа, как правило, более доходные и менее рисковые, чем венчурные фонды. Также ВО фонды имеют меньшую подверженность системному риску, чем рынок, а VC фонды - больше, чем рынок.

Таким образом, формулируемые гипотезы относительно доходности и риска выглядят следующим образом:

Гипотеза 1. Медиана меньше среднего значения показателей доходности ФПИ и меньше среднего значения доходности рыночных эквивалентов сделок.

Верность данного утверждения указывает на то, что большинство фондов проигрывает рынку.

Гипотеза 2. Среднее значение показателей доходности ФПИ больше среднего значения доходности рыночных эквивалентов.

Верность данного утверждения указывает на то, что существуют фонды, которые обеспечивают доходность значительно выше рыночной.

Гипотеза 3. Среднее значение показателей доходности $B O$ фондов больше среднего значения для $V C$ фондов.

Верность данного утверждения указывает на то, что фонды выкупа более эффективные, чем венчурные фонды.

Гипотеза 4. Стандартное отклонение доходности ФПИ выше стандартного отклонения доходности рыночных эквивалентов.

Верность данного утверждения указывает на то, что риск ФПИ выше рыночного.

Гипотеза 5. Бета для ВО меньше 1.

Верность данного утверждения указывает на то, что системный риск фондов выкупа ниже рыночного.

Гипотеза 6. Бета для $V C$ больше 1.

Верность данного утверждения указывает на то, что системный риск фондов выше рыночного.

Исходя из того что срок существования фонда составляет 10 лет и средняя продолжительность владения одним активом для ФПИ должна составлять 5 лет или несколько больше (с учетом увеличения срока жизни фонда до 12 лет).

Гипотеза 7. HP $\geq 5$.

Верность данного утверждения указывает на то, что инвестиционный период данной выборки сделок в среднем соответствует практике фондов развитых стран.

Гипотезы будут проверяться на основе описательной статистики выборки. Описательная статистика представляет собой оценку таких параметров выборки как среднее, медиана, стандартное отклонение, асимметрия и эксцесс для основных показателей выборки. Для определения подверженности класса активов системному риску будет также рассчитан коэффициент бета. Далее полученные параметры сопоставляются с результатами других 
исследований для подтверждения адекватности данных. Затем будут рассмотрены различия оценок параметров между странами. Данный этап исследования предполагает качественную оценку основных характеристик инвестиций в ФПИ, однако не оценивает взаимозависимость показателей между собой и значимость оценок.

\section{Источники данных}

За основу исследования была взята база данных аналитического агентства Preqin. Конечная выборка была проверена и дополнена данными агентств ACJV, GVCEPE, Preqveca, Venture database (см. таблицу 3). Первичными источниками информации являются данные управляющих партнеров, инвесторов, а также информация из СМИ.

Таблица 3

\section{Источники данных}

\begin{tabular}{|c|c|c|c|c|}
\hline \multirow{2}{*}{ Страна } & \multirow{3}{|c|}{ Количество наблюдений } \\
\cline { 2 - 5 } & Источник данных & ВО & VC & Всего \\
Китай & Preqin, ACJV database & 509 & 337 & 846 \\
\hline Индия & Preqin, ACJV database & 464 & 183 & 647 \\
\hline Бразилия & Preqin, GVCEPE & 187 & 178 & 365 \\
\hline Россия и СНГ & Preqin, Preqveca, Venture database & 78 & 21 & 99 \\
\hline Всего & & 1238 & 719 & 1957 \\
\hline
\end{tabular}

Выборка представляет собой информацию о завершенных парах сделок покупки и продажи портфельных компаний среди фондов прямых инвестиций на территории Китая, Индии, Бразилии, России и некоторых стран СНГ в период с 2000 по 2012 г., в привязке к году продажи. Выборка содержит 1957 наблюдений, из которых 719 сделок отнесены к сделкам венчурного финансирования (VC), а 1238 (BO) - к сделкам по выкупу. Каждая пара сделок характеризуется наименованием инвестора (управляющего фонда), наименованием портфельной компании, суммой приобретения (size) и годом приобретения (enter year), cyммой продажи (exit price) и годом продажи актива (exit year), возрастом управляющей компании (age), номером фонда (seq), страной, а также является ли фонд венчурным или фондом выкупа (type). При отсутствии части необходимых данных или возникновении противоречащих друг другу данных в процессе сопоставлении информации из разных источников наблюдение исключалось из выборки.

На основе имеющихся данных дополнительно для каждой сделки были рассчитаны следующие показатели:

- $\quad$ Инвестиционный период (HP - holding period) - разница между годом продажи и годом покупки.

- Инвестиционный мультипликатор (IM-investment multiple) - рост стоимости актива в течение инвестиционного периода (см. раздел «Методология»).

- Среднегодовая доходность сделки (CAR-compound annual return) - среднегодовой рост стоимости актива в течение инвестиционного периода (см. раздел «Методология»).

- $\quad$ Инвестиционный мультипликатор для рыночного эквивалента сделки (IMME - investment multiple of market equivalent) - рост индекса фондовой биржи в данной стране в течение инвестиционного периода (см. раздел «Методология»).

- $\quad$ Среднегодовая доходность рыночного эквивалента сделки (CARME - compound annual return of market equivalent) - среднегодовой рост индекса фондовой биржи в данной стране в течение инвестиционного периода (см. раздел «Методология»).

Достоинством такой выборки является значительный временной и географический охват, а также высокая надежность наблюдений, обеспеченная перекрестной проверкой данных.

Недостатком выборки является малое количество наблюдений для Бразилии, России и СНГ, 
а также риск завышения показателей доходности ввиду отсутствия стандартов требований по раскрытию информации для управляющих фондов. Значительное число сделок было исключено из выборки из-за отсутствия данных о сумме продажи актива, что может являться следствием нежелания управляющих фондами раскрывать негативный результат.

\section{Общее описание параметров выборки}

Для описания основных показателей полученной выборки были рассчитаны максимальное и минимальное значения, медиана, среднее, стандартное отклонение, асимметрия (распределение 3 момента), эксцесс (распределение 4 момента). Результаты расчетов представлены в таблице 4.

Таблица 4

Характеристики переменных выборки

\begin{tabular}{|c|c|c|c|c|c|c|}
\hline Характеристика & $I M$ & CAR (\%) & $H P$ & Size & Age & Seq \\
\hline Макс. & 16,78 & $1135 \%$ & 23,00 & 530,00 & 36,00 & 10,00 \\
\hline Мин. & 0,00 & $-100 \%$ & 1,00 & 0,04 & 1,00 & 1,00 \\
\hline Медиана & 1,22 & $7 \%$ & 3,00 & 19,00 & 9,00 & 2,00 \\
\hline Среднее & 2,05 & $26 \%$ & 3,27 & 36,26 & 11,11 & 2,42 \\
\hline Стандартное откл. & 2,04 & $98 \%$ & 2,15 & 51,80 & 8,45 & 1,64 \\
\hline Асимметрия & 2,25 & 5,38 & 1,19 & 5,24 & 1,09 & 2,07 \\
\hline Эксцесс & 13,16 & 64,3 & 7,99 & 44,72 & 6,56 & 11,15 \\
\hline
\end{tabular}

Показатели доходности по сделкам варьируются в широком интервале от 0 (полное списание средств) до 16,78 раз увеличения стоимости актива, и от $-100 \%$ до $1135 \%$ среднегодовой доходности. Средние и медианные значения соответственно равны 1,22 и 2,05 для инвестиционного мультипликатора, $7 \%$ и 26\% для среднегодовой доходности. Средневзвешенные по размеру инвестиций показатели доходности $\mathrm{IM}=2,26$, a CAR = 41\%. Оба распределения имеют большое стандартное отклонение: 2,04 и 98\%, что указывает на низкую однородность распределения. Показатель асимметрии больше нуля для обоих показателей указывает на то, что вершина смещена в сторону низких значений, о чем также свидетельствует разница между средним и медианным значениями. Однако, так как распределения являются островершинными (показатель эксцесса больше 3), а значит, обладают «тяжелыми хвостами» (большим количеством выбросов из общей тенденции), вывод об ожидаемой доходности на основе наблюдаемых средних и медианных значений не может быть надежным. Следует отметить, что высокая волатильность доходности сделок не является непосредственной характеристикой измерения риска для инвесторов, так как упускается из виду эффект диверсификации, который достигается при рассмотрении пула сделок в рамках одного фонда. Таким образом, при сопоставлении полученных результатов доходности и инвестиционного мультипликатора для отдельных сделок с аналогичными исследованиями среди фондов развитых стран очевидна значительная волатильность показателя доходности CAR, который является прокси-показателем для IRR. Вместе с тем медиана для всех исследований значительно превышает среднее значение, что подтверждает предпосылку о положительно смещенном распределении доходности. Также следует отметить, что при отсутствии временного фактора отличия в оценках доходности становятся менее заметными и дисперсия оценок падает (см. табл. 5).

Таблица 5

Сравнения параметров распределения с аналогичными исследованиями

\begin{tabular}{|c|c|c|c|c|}
\hline Исследование & $\begin{array}{c}\text { Harris, Jenkinson, } \\
\text { Kaplan (2010) }\end{array}$ & Kaplan, Schoar (2005) & $\begin{array}{c}\text { Nowak, Knigge, Shmidt } \\
(2004)\end{array}$ & $\begin{array}{c}\text { Имеющаяся } \\
\text { выборка }\end{array}$ \\
\hline $\begin{array}{c}\text { Временной интервал } \\
\text { (годы начала } \\
\text { инвестирования) }\end{array}$ & $1984-2008$ гг. & $1995-2005$ гг. & $1971-1999$ гг. & $1990-2012$ гг. \\
\hline
\end{tabular}




\begin{tabular}{|c|c|c|c|c|}
\hline География & $\begin{array}{c}\text { Страны с развитой } \\
\text { экономикой }\end{array}$ & $\begin{array}{c}\text { Страны с развитой } \\
\text { экономикой }\end{array}$ & США, Европа & BRIC \\
\hline Методика & $\begin{array}{c}\text { Валовая доходность, } \\
\text { на основе денежных } \\
\text { потоков }\end{array}$ & $\begin{array}{c}\text { Валовая доходность, } \\
\text { на основе денежных } \\
\text { потоков }\end{array}$ & $\begin{array}{c}\text { Валовая доходность, } \\
\text { на основе денежных } \\
\text { потоков }\end{array}$ & $\begin{array}{c}\text { Валовая доходность, } \\
\text { на основе } \\
\text { совершенных сделок }\end{array}$ \\
\hline Количество наблюдений & 729 (фондов) & 746 (фондов) & 70 (фондов) & 1957 (сделок) \\
\hline Медиана (IRR/CAR) & $10 \%$ & $12 \%$ & $12 \%$ & $7 \%$ \\
\hline Среднее (IRR/CAR) & $17 \%$ & $17 \%$ & $47 \%$ & $26 \%$ \\
\hline $\begin{array}{c}\text { Стандартное } \\
\text { отклоненине (IRR/CAR) }\end{array}$ & $39 \%$ & $32 \%$ & $56 \%$ & $98 \%$ \\
\hline Медиана (IM) & 1,10 & 1,66 & 1,32 & 1,22 \\
\hline Средннее (IM) & 1,70 & 2,24 & 2,70 & 2,05 \\
\hline $\begin{array}{c}\text { Стандартное } \\
\text { отклоненине (IM) }\end{array}$ & 1,56 & 2,30 & 2,10 & 2,04 \\
\hline
\end{tabular}

Остальные показатели выборки характеризуются похожими свойствами: большая дисперсия, положительно смещенное остроконечное распределение. Следует отметить, что возраст управляющей компании и инвестиционный период и номер фонда для сделки более однородны, о чем говорят более низкие показатели стандартного отклонения (меньше среднего значения) и разница между медианой и средним значением. Большая однородность возраста управляющей компании частично объясняется тем, что в выборке присутствует только 529 уникальных наблюдений, остальные повторяются, в случае если у одной управляющей компании несколько сделок. Количество повторений варьируется от 1 до 33 раз. Эксцесс распределения возраста управляющей компании составляет 6,33, что является наиболее низким показателем среди основных параметров. Сумма приобретения компании, наоборот, является наиболее волатильным показателем со средним и медианным значением \$36,2 млн и \$19 млн и наиболее ярко выраженным положительным смещением.

Положительный коэффициент асимметрии распределения возраста, номера фонда и суммы приобретения указывает на наличие в выборке преобладающего количества молодых управляющих компаний, компаний с малым опытом инвестирования, а также и относительно небольшой размер сделок.

\section{Сравнение оценок параметров между странами}

Для выявления географических различий были рассчитаны медианные и средние значения (первое и второе значения в каждом столбце) и стандартное отклонение параметра (третье значение) отдельно для ВО и VC фондов в каждой стране.

Таблица 7

Сравнение характеристик переменных между странами и типами фондов

\begin{tabular}{|c|c|c|c|c|c|c|c|c|}
\hline \multirow{2}{*}{ Переменная } & \multicolumn{2}{c}{ Китай } & \multicolumn{3}{c}{ Индия } & \multicolumn{2}{c|}{ Бразилия } & \multicolumn{2}{c|}{ Россия + СНГ } \\
\cline { 2 - 9 } & $\mathbf{B O}$ & $\mathbf{V C}$ & $\mathbf{B O}$ & $\mathbf{V C}$ & $\mathbf{B O}$ & $\mathbf{V C}$ & $\mathbf{B O}$ & $\mathbf{V C}$ \\
\cline { 2 - 9 } & 1,47 & 1,23 & 0,98 & 1,10 & 1,01 & 1,02 & 2,36 & 2,05 \\
& 2,11 & 1,97 & 2,07 & 2,30 & 1,72 & 1,75 & 2,74 & 2,40 \\
$\boldsymbol{I M M E}$ & $(2,03)$ & $(1,82)$ & $(2,18)$ & $(2,41)$ & $(1,7)$ & $(1,61)$ & $(2,51)$ & $(2,41)$ \\
& 1,49 & 1,39 & 1,36 & 1,48 & 1,40 & 1,26 & 1,25 & 1,35 \\
$\boldsymbol{C A R}$ & 1,53 & 1,48 & 1,65 & 1,52 & 1,72 & 1,58 & 1,35 & 1,45 \\
& $(0,80)$ & $(0,90)$ & $(1,03)$ & $(0,98)$ & $(0,91)$ & $(1,13)$ & $(1,25)$ & $(0,99)$ \\
\cline { 2 - 9 } & 0,12 & 0,07 & $-0,01$ & 0,02 & 0,01 & 0,01 & 0,22 & 0,21 \\
& 0,27 & 0,20 & 0,31 & 0,28 & 0,28 & 0,24 & 0,13 & 0,34 \\
& $(0,89)$ & $(0,81)$ & $(1,21)$ & $(0,98)$ & $(1,00)$ & $(0,83)$ & $(0,90)$ & $(1,20)$
\end{tabular}




\begin{tabular}{|c|c|c|c|c|c|c|c|c|}
\hline CARME & $\begin{array}{c}0,11 \\
0,13 \\
(0,12)\end{array}$ & $\begin{array}{c}0,10 \\
0,12 \\
(0,08)\end{array}$ & $\begin{array}{c}0,15 \\
0,14 \\
(0,16)\end{array}$ & $\begin{array}{c}0,14 \\
0,13 \\
(0,15)\end{array}$ & $\begin{array}{c}0,13 \\
0,15 \\
(0,13)\end{array}$ & $\begin{array}{c}0,15 \\
0,13 \\
(0,10)\end{array}$ & $\begin{array}{c}0,09 \\
0,10 \\
(0,15)\end{array}$ & $\begin{array}{c}0,06 \\
0,08 \\
(0,19)\end{array}$ \\
\hline$\beta$ & 0,52 & 0,91 & 0,49 & 1,19 & 0,62 & 1,00 & $-0,05$ & 0,10 \\
\hline & 48,03 & 15,52 & 24,79 & 7,08 & 22,73 & 8,78 & 14,00 & 1,00 \\
\hline Size & $\begin{array}{c}77,68 \\
(78,64)\end{array}$ & $\begin{array}{c}18,05 \\
(12,97)\end{array}$ & $\begin{array}{c}30,75 \\
(24,91)\end{array}$ & $\begin{array}{c}8,07 \\
(6,39)\end{array}$ & $\begin{array}{c}26,91 \\
(20,94)\end{array}$ & $\begin{array}{l}11,53 \\
(9,4)\end{array}$ & $\begin{array}{c}31,79 \\
(68,19)\end{array}$ & $\begin{array}{c}1,28 \\
(0,83)\end{array}$ \\
\hline & 3,00 & 3,00 & 3,00 & 3,00 & 2,00 & 2,00 & 4,00 & 2,00 \\
\hline$H P$ & $\begin{array}{c}3,41 \\
(1,88)\end{array}$ & $\begin{array}{c}3,24 \\
(1,70)\end{array}$ & $\begin{array}{c}3,36 \\
(2,37)\end{array}$ & $\begin{array}{c}3,61 \\
(2,48)\end{array}$ & $\begin{array}{c}2,48 \\
(1,88)\end{array}$ & $\begin{array}{c}2,76 \\
(2,01)\end{array}$ & $\begin{array}{c}4,37 \\
(3,15)\end{array}$ & $\begin{array}{c}3,00 \\
(2,37)\end{array}$ \\
\hline Age & $\begin{array}{c}11,00 \\
12,52 \\
(9,04)\end{array}$ & $\begin{array}{l}11,00 \\
12,41 \\
(9,26)\end{array}$ & $\begin{array}{c}11,00 \\
11,77 \\
(8,84)\end{array}$ & $\begin{array}{l}11,00 \\
12,04 \\
(9,08)\end{array}$ & $\begin{array}{c}6,00 \\
6,60 \\
(4,40)\end{array}$ & $\begin{array}{c}7,00 \\
7,64 \\
(4,53)\end{array}$ & $\begin{array}{c}7,50 \\
9,40 \\
(5,36)\end{array}$ & $\begin{array}{c}8,00 \\
9,05 \\
(5,61)\end{array}$ \\
\hline Seq & $\begin{array}{c}2,00 \\
2,66 \\
(1,81) \\
\end{array}$ & $\begin{array}{c}2,00 \\
2,60 \\
(1,73)\end{array}$ & $\begin{array}{c}2,00 \\
2,56 \\
(1,62) \\
\end{array}$ & $\begin{array}{c}2,00 \\
2,63 \\
(1,79) \\
\end{array}$ & $\begin{array}{c}2,00 \\
2,02 \\
(1,25)\end{array}$ & $\begin{array}{c}1,00 \\
1,76 \\
(1,11)\end{array}$ & $\begin{array}{c}1,00 \\
1,51 \\
(0,82)\end{array}$ & $\begin{array}{c}1,00 \\
1,24 \\
(0,44) \\
\end{array}$ \\
\hline
\end{tabular}

Показатели доходности, рассчитанные отдельно для разных стран и стратегий инвестирования, подтверждают выводы, сделанные по выборке в целом: высокая дисперсия доходности сделок и положительное смещение. На это указывают наблюдаемые значения стандартного отклонения и положительная разность среднего и медианы (за исключением CAR для BO сделок на рынке России и СНГ).

Наиболее высокие показатели доходности демонстрирует рынок России и СНГ, однако он же имеет наименьшее количество наблюдений и самую высокую дисперсию, что указывает на ненадежность полученных оценок (IM для ВО и VC равен 2,36 и 2,05; CAR для ВО и VC равен $22 \%$ и 21\%). Вторым по доходности, ориентируясь на медианные показатели, идет рынок Китая (IM для ВО и VC равен 1,47 и 1,23; CAR для ВО и VC равен 12\% и 7\%), который имеет наиболее низкое стандартное отклонение и наибольшее количество наблюдений, а значит, большую надежность оценок. Индия и Бразилия имеют медианную среднегодовую доходность близкую к нулю и наиболее значительное расхождение между средним и медианой, что говорит о значительном смещении доходности в сторону низких значений.

При сравнении показателей доходности разных типов фондов между собой, а также с рыночными эквивалентами, можно проанализировать ряд ранее выдвинутых гипотез. Так, например, подтверждаются гипотезы о том, что большая часть фондов проигрывает рынку (гипотеза № 1, подтверждается для всех стран, кроме России), а также о том, что существует часть фондов, которые существенно опережают рыночную доходность (гипотеза № 2, подтверждается для всех рынков). Предположение о большей эффективности фондов выкупа по сравнению с венчурными фондами (гипотеза № 3) подтвердилось только на рынках Китая и России, а для рынков Индии и Бразилии результаты обратные. Предположение о большем по сравнению с рынком риском инвестиций ФПИ (гипотеза № 4) подтверждено для всех стран. Также подтверждена гипотеза о том, что системный риск фондов выкупа ниже рыночного (гипотеза № 5). Вместе с тем гипотеза о том, что венчурные фонды подвержены системному риску больше рынка (гипотеза № 6) нашла только частичное подтверждение, так как Россия и Китай продемонстрировали обратный результат.

Оценки остальных параметров фондов также подтверждают предположение о низкой однородности и положительном смещении распределений для выборки в целом, демонстрируя высокую дисперсию и превышение медианы над средними для всех параметрами.

На основе наблюдаемых значений рынок Китая является наиболее зрелым, крупным рынком, так как имеет наибольший средний возраст управляющей компании $(12,52$ и 12,41 года для ВО и VC), размер сделок (\$48,03 млн для ВО, $\$ 15,52$ млн для VC), а также количество открытых фондов (на одного управляющего приходится 2-2,5 фонда). 
Близкие значения показателей по суммам сделок демонстрируют рынки Бразилии и Индии (\$24,79 млн и \$22,73 млн для сделок ВО и \$7,08 млн и \$8,78 млн VC), однако управляющие фондами Индии значительно старше бразильских (11 против 7 лет) и имеют в среднем больше открытых фондов (2,56 против 2,02 для ВО и 2,63 против 1,76 для VC). Кроме того, стратегии двух рынков отличаются периодом инвестирования (3 и 2 года соответственно).

Наиболее неразвитым среди представленных рынков представляется российский. Он уступает по сумме сделок ( $\$ 14$ млн и \$1 млн - медианные значения для ВО и VC), имеет наименьшее количество открытых фондов (1 для ВО и 1 для $\mathrm{VC}$ ) и обладает наиболее молодыми управляющими после Бразилии (7,5 и 8 лет для $\mathrm{BO}$ и VC).

Таким образом, локальные рынки имеют существенные различия в наблюдаемых средних и медианных значениях параметров, и их условно можно ранжировать по степени «развитости». В то же время все четыре рынка обладают схожей формой распределений основных характеристик, с медианным значением, превышающим среднее.

Различия наблюдаемых медианных, средних значений и стандартного отклонения параметров выборки между фондами выкупа и венчурными фондами не дают однозначного ответа на вопрос о различии фондов. Показатели доходности, периода инвестирования, возраста управляющих и количества открытых фондов имеют либо близкие значения, либо различия, которые не подтверждаются на остальных рассматриваемых рынках. Стабильным существенным различием между ВО и VC фондами для всех рынков является сумма сделки. Кроме того, следует отметить, что предположение о продолжительности инвестиционного периода (гипотеза № 7, НР >5) не подтвердилась ни на одном из рынков, и средний период инвестирования составил 3,3 года.

Обобщая все вышесказанное, описательная статистика выборки и анализ распределения показателей доходности позволяют сделать следующие выводы:

1. Было выявлено, что инвестиционный профиль ФПИ развивающихся стран имеет те же характеристики, что и ФПИ развитых стран, за исключением нескольких пунктов (см. табл. 8). Также было сделан ряд наблюдений за рамками выдвинутых гипотез.

2. Распределение всех переменных выборки имеет значительную дисперсию, положительное смещение и островершинную форму. Это говорит о том, что основная масса наблюдений представлена сделками с доходностью, возрастом управляющих компаний, размером первоначальных инвестиций и периодом инвестирования меньше среднего значения, однако имеет большое количество выбросов. Данная форма распределения соответствует результатам исследований на рынках развитых стран, что подтверждает адекватность данных.

3. Наиболее доходным рынком является российский, хотя он имеет наименьшую надежность оценок. За ним следуют по очереди рынки Китая, Индии и Бразилии.

4. Рынки условно можно ранжировать по степени развитости, взяв за основу возраст управляющих компаний, количество открытых фондов и размер сделок. Таким образом, наиболее развитым рынком является Китай, за ним следует рынок Индии, потом - Бразилии и России.

5. Существует положительная корреляция между возрастом компании и номером фонда сделки, что подтверждает адекватность данных.

\begin{tabular}{|c|c|c|c|}
\hline $\begin{array}{c}\text { Номер } \\
\text { гипотезы }\end{array}$ & Гипотеза & Значение & Описательная статистика \\
\hline 1 & $\begin{array}{c}\text { Мед. (Дох. ФПИ) }<\text { Ср. знач. (Дох. ФПИ); } \\
\text { Мед. (Дох. ФПИ) }<\text { Ср. знач. (Дох. рынка) }\end{array}$ & $\begin{array}{c}\text { Большинство фондов } \\
\text { проигрывает рынку }\end{array}$ & $\begin{array}{c}\text { Справедливо для всех рынков кроме } \\
\text { России }\end{array}$ \\
\hline
\end{tabular}




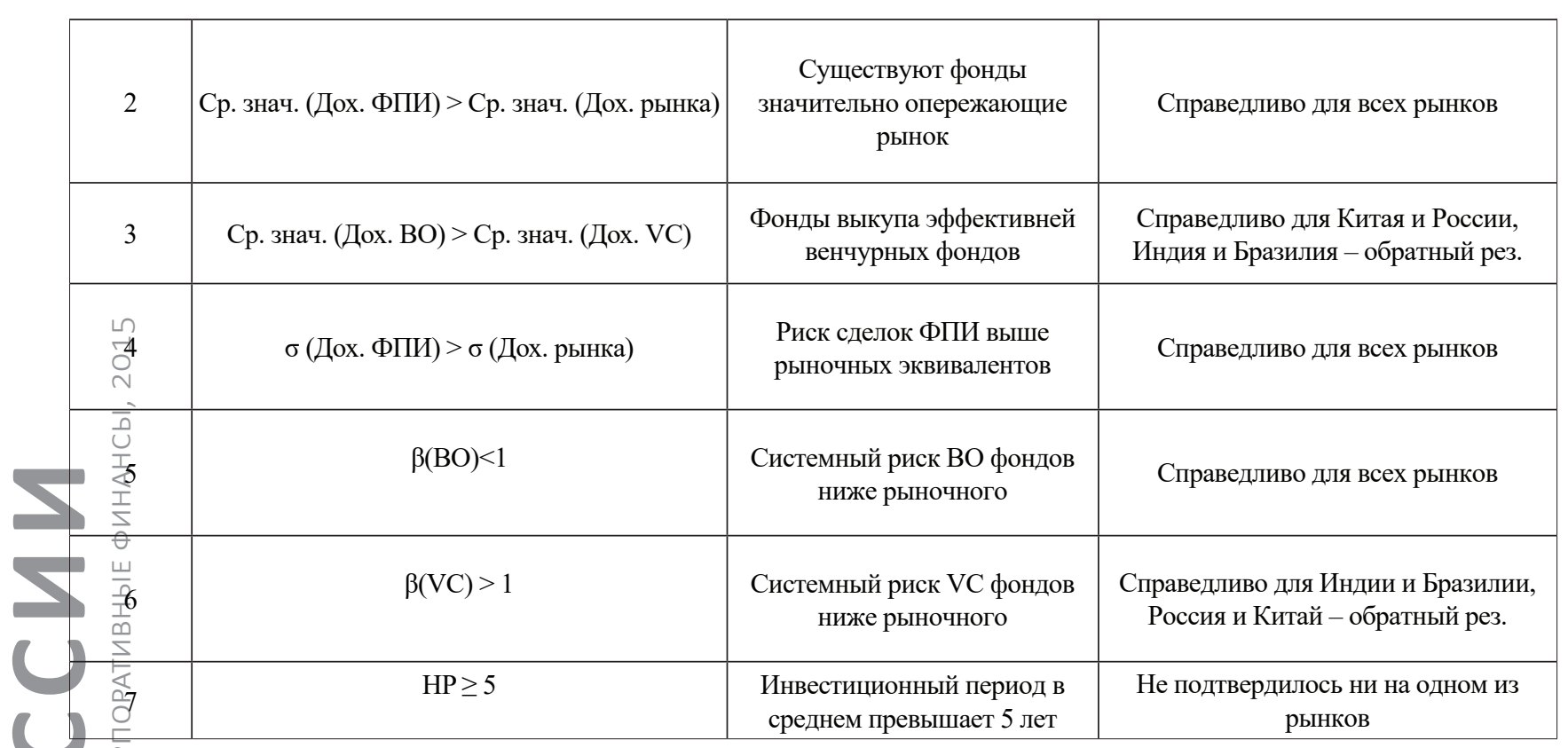

\section{Выводы}

Из-за отсутствия данных о денежных потоках оценить эффективность ФПИ для развивающихся рынков капитала представляется более сложной задачей, чем для развитых, так как использование традиционных показателей доходности TVPI и IRR невозможно.

В данном исследовании был предложен подход, предполагающий применение скорректированных показателей доходности для ФПИ, который может быть реализован без использования данных о денежных потоках и чистых активов фондов. Предложенные показатели могут быть рассчитаны на основе имеющихся в публичном доступе данных о портфельных сделках фондов.

В исследовании была представлена методология анализа эффективности ФПИ на основе портфельных сделок, а также проведен анализ эмпирических данных на выборке из 1957 сделок стран BRIC в период с 2000 по 2012 г.

Результаты эмпирического анализа в целом подтверждают ряд фундаментальных характеристик ФПИ, ранее выявленных для развитых рынков капитала, таких как:

1. Сделки ФПИ в развивающихся странах являются более рисковыми активами, чем традиционные инструменты.

2. Доходность у большинства сделок ниже доходности фондового рынка, однако наиболее успешные существенно опережают рынок.

3. Инвестиции фондов выкупа имеют коэффициент $\beta$ меньше единицы, что говорит о малой подверженности системному риску.

Некоторые характеристики нашли подтверждение только частично:

Инвестиции венчурных фондов имеют коэффициент $\beta$ больше единицы для рынков Бразилии и Индии и меньше единицы для России и Китая.

Доходность инвестиций ВО фондов выше доходности VC фондов в России и Китае. В Индии и Бразилии - результат обратный.

Оставшаяся часть характеристик принципиально отличается от выявленных на развитых рынках капитала:

Период владения инвестициями для ФПИ развивающихся стран меньше, чем для развитых стран и в среднем составляет 3,3 года. 


\section{Список литературы}

1. Chen, P., Baierl, G., and Kaplan, P. (2002), "Venture capital and its role in strategic asset allocation”, Journal of Portfolio Management, vol. 28, no. 2, pp, 83-90.

2. Cochrane, J.H. (2005), "Financial Markets and the Real Economy", Foundations and Trends $(R)$ in Finance, now publishers, vol. 1, no. 1, pp. 1-101

3. Conroy, R.M., and Harris R.S. (2007), “How Good Are Private Equity Returns?” Journal of Applied Corporate Finance, no. 9, pp. 96-108.

4. Cressy, R., Munari, F. and Malipiero, A. (2007), "Playing to their strengths? Evidence that specialization in the private equity industry confers competitive advantage", Journal of Corporate Finance, Elsevier, vol. 13, no. 4, pp. 647-669.

5. Cumming, D., and Walz, U. (2010), "Private equity returns and disclosure around the world", Journal of International Business Studies, vol. 41, no. 4, pp. 727-755.

6. Diller, Ch, and Kaserer, Ch. (2004), "European private equity funds: A cash flow based performance analysis", CEFS Working Paper Series 2004-01, Center for Entrepreneurial and Financial Studies (CEFS), Technische Universität München.

7. Driessen, J., Lin, T.-C., and Phalippou, L. (2008), “A new method to estimate risk and return of non-traded assets from cash flows: The case of private equity funds". NBER Working Paper Series.

8. Ellis, C., and Steer, J. (2011), “Are UK venture capital and private equity valuations overoptimistic?”, BVCA Research Report, April.

9. Gompers, P., Kovner, A., and Lerner, J. (2009), "Specialization and success: Evidence from venture capital", Journal of Economics and Management Strategy, vol. 18, no. 3, pp. 817844.

10. Gompers, P., and Lerner, J. (1999), Capital Market Imperfections in Venture Markets: A Report to the Advanced Technology Program. Washington: Advanced Technology Program, U.S. Department of Commerce.

11. Gottschalg, O. (2010), “The real reasons to invest in private equity”. HEC Paris. Working Paper.

12. Groh, A.P., and Gottschalg, O. (2008), "Measuring the risk-adjusted performance of US buyouts". NBER Working Paper No. W14148.

13. Hege, U., Schwienbacher, A., and Palomino, F. (2009), "Venture Capital Performance: The Disparity between Europe and the United States", Revue Finance, vol. 30, no. 1, pp. 7-50.

14. Hwang, M., Quigley, J., and Woodward, S. (2005), An Index for Venture Capital, 1987-2003, Contributions to Economic Analysis \& Policy 4, 1-43.

15. Jääskeläinen, J. (2011), Operational performance of Nordic private equity backed buyouts in the recession of 2007-2009. Aalto University School of Economics, Department of Finance. Master's thesis in Finance.

16. Jones, C., and Rhodes-Kropf, M. (2003), "The price of diversifiable risk in venture capital and private equity". AFA 2003 Washington, DC Meetings. Working Paper.

17. Kaplan, S.N., Martel, F., and Strömberg, P. (2003), "How do legal differences and learning affect financial contracts?", Journal of Financial Intermediation, vol. 16, no. 3, pp. 273-311.

18. Kaplan, S.N., and Schoar, A. (2005), "Private equity performance: Returns, persistence and capital flows", Journal of Finance, vol. 60, no. 4, pp. 1791-1823.

19. Kaplan, S.N., and Strömberg, P. (2009), "Leveraged buyouts and private equity", Journal of Economic Perspectives, vol, 23, no. 1, pp. 121-146.

20. Ljungqvist, A., and Richardson, M. (2003), "The cash flow, return and risk characteristics of private equity". NYU Working Paper No. FIN-03-001. 
21. Long, A., and Nickels, C. (1996), “A private investment benchmark”, mimeo; paper presented to AIMR Conference on Venture Capital Investing, February.

22. Metrick, A., and Yasuda, A. (2010), "The economics of private equity funds", Review of Financial Studies, vol. 23, no. 6, pp. 2303-2341.

23. Manigart, S., Joos, P., and De Vos, D. (1994), "The performance of publicly traded European venture capital companies”, Journal of Small Business Finance, vol. 3, no. 2, pp.111-125.

24. Meggison, W.L., Nash, R.C., Netter, J.M., and Poulsen, A.B. (2004), “The Choice of Private Versus Public Capital Markets: Evidence from Privatizations", The Journal of Finance, vol. 59, no. 6, pp 2835-2870.

25. Nikoskelainen, E., and Wright, M. (2007), "The impact of corporate governance mechanisms on value increase in leveraged buyouts", Journal of Corporate Finance, vol. 13, no. 4, pp. 511-537.

26. Schmidt, D. (2006), "Private equity versus stocks: Do the alternative asset's risk and return characteristics add value to the portfolio?", Journal of Alternative Investments, vol. 9, no. 1, pp. 28-47.

27. Schmidt, D., Nowak, E., and Knigge, A. "On the Performance of Private Equity Investments: Does Market Timing Matter?” Working Paper, 2004.

28. Phalippou, L., and Gottschalg, O. (2009), "The performance of private equity funds" Review of Financial Studies, vol. 22, no. 4, pp. 1747-1776.

29. Phalippou, L., and Zollo, M. (2005), "What Drives Private Equity Fund Performance?", SSRN working paper. 
PRIVATE EQUITY PERFORMANCE IN EMERGING MARKETS

Ivan Rodionov,

professor of "Economics and Finance” Higher School of Economics

Stepan Gusamov,

postgraduate student

National Research University Higher School of Economics

\begin{abstract}
Because of the lack of data on cash flows, it is impossible to use traditional measures of return such as IRR and TVPI for evaluation the performance of private equity funds in emerging markets.

In this study, we proposed an approach based on the use of adjusted rates of return for the PE funds, which can be implemented without the use of data on cash flows and net assets of the funds. The proposed indicators can be calculated on the basis of the publicly available data on portfolio transactions of the fund.

The study was presented methodology based on the performance of private equity portfolio transactions as well as the analysis of empirical data on a sample of 1957 deals in BRIC countries from 2000 to 2012.
\end{abstract}

The results of the empirical analysis largely support a number of fundamental characteristics of the PE funds, previously identified for the developed capital markets such as:

1. Private equity deals in developing countries are more risky assets than traditional instruments.

2. The return on the majority of transactions is below the return of the stock market, however, the most successful are significantly ahead of the market.

3. Coefficient $\beta$ of buyout funds is less than one, indicating the low exposure to systemic risk.

Some characteristics were confirmed only in part:

1. The investments of venture capital funds have a coefficient $\beta$ is greater than one for the markets of Brazil and India, and less than one for Russia and China.

2. Return on investment is higher for buyout funds than for venture capital funds in Russia and China. In India and Brazil - the opposite result.

The rest of the characteristics are fundamentally different from the identified in the developed capital markets:

1. The period of ownership for the private equity fund investment in developing countries is less than for developed countries and is an average of 3.3 years.

Keywords: private equity, venture capital, asset valuation, performance

JEL: G12, G23

\title{
References
}

1. Chen, P., Baierl, G., and Kaplan, P. (2002), "Venture capital and its role in strategic asset allocation”, Journal of Portfolio Management, vol. 28, no. 2, pp, 83-90.

2. Cochrane, J.H. (2005), "Financial Markets and the Real Economy", Foundations and Trends $(R)$ in Finance, now publishers, vol. 1, no. 1, pp. 1-101

3. Conroy, R.M., and Harris R.S. (2007), "How Good Are Private Equity Returns?” Journal of Applied Corporate Finance, no. 9, pp. 96-108.

4. Cressy, R., Munari, F. and Malipiero, A. (2007), "Playing to their strengths? Evidence that specialization in the private equity industry confers competitive advantage", Journal of Corporate Finance, Elsevier, vol. 13, no. 4, pp. 647-669. 
5. Cumming, D., and Walz, U. (2010), "Private equity returns and disclosure around the world", Journal of International Business Studies, vol. 41, no. 4, pp. 727-755.

6. Diller, Ch, and Kaserer, Ch. (2004), "European private equity funds: A cash flow based performance analysis", CEFS Working Paper Series 2004-01, Center for Entrepreneurial and Financial Studies (CEFS), Technische Universität München.

7. Driessen, J., Lin, T.-C., and Phalippou, L. (2008), “A new method to estimate risk and return of non-traded assets from cash flows: The case of private equity funds". NBER Working Paper Series.

8. Ellis, C., and Steer, J. (2011), “Are UK venture capital and private equity valuations overoptimistic?”, BVCA Research Report, April.

9. Gompers, P., Kovner, A., and Lerner, J. (2009), "Specialization and success: Evidence from venture capital", Journal of Economics and Management Strategy, vol. 18, no. 3, pp. 817844.

10. Gompers, P., and Lerner, J. (1999), Capital Market Imperfections in Venture Markets: A Report to the Advanced Technology Program. Washington: Advanced Technology Program, U.S. Department of Commerce.

11. Gottschalg, O. (2010), “The real reasons to invest in private equity”. HEC Paris. Working Paper.

12. Groh, A.P., and Gottschalg, O. (2008), "Measuring the risk-adjusted performance of US buyouts". NBER Working Paper No. W14148.

13. Hege, U., Schwienbacher, A., and Palomino, F. (2009), "Venture Capital Performance: The Disparity between Europe and the United States", Revue Finance, vol. 30, no. 1, pp. 7-50.

14. Hwang, M., Quigley, J., and Woodward, S. (2005), An Index for Venture Capital, 1987-2003, Contributions to Economic Analysis \& Policy 4, 1-43.

15. Jääskeläinen, J. (2011), Operational performance of Nordic private equity backed buyouts in the recession of 2007-2009. Aalto University School of Economics, Department of Finance. Master's thesis in Finance.

16. Jones, C., and Rhodes-Kropf, M. (2003), "The price of diversifiable risk in venture capital and private equity”. AFA 2003 Washington, DC Meetings. Working Paper.

17. Kaplan, S.N., Martel, F., and Strömberg, P. (2003), "How do legal differences and learning affect financial contracts?", Journal of Financial Intermediation, vol. 16, no. 3, pp. 273-311.

18. Kaplan, S.N., and Schoar, A. (2005), "Private equity performance: Returns, persistence and capital flows", Journal of Finance, vol. 60, no. 4, pp. 1791-1823.

19. Kaplan, S.N., and Strömberg, P. (2009), "Leveraged buyouts and private equity", Journal of Economic Perspectives, vol, 23, no. 1, pp. 121-146.

20. Ljungqvist, A., and Richardson, M. (2003), "The cash flow, return and risk characteristics of private equity". NYU Working Paper No. FIN-03-001.

21. Long, A., and Nickels, C. (1996), “A private investment benchmark”, mimeo; paper presented to AIMR Conference on Venture Capital Investing, February.

22. Metrick, A., and Yasuda, A. (2010), "The economics of private equity funds", Review of Financial Studies, vol. 23, no. 6, pp. 2303-2341.

23. Manigart, S., Joos, P., and De Vos, D. (1994), "The performance of publicly traded European venture capital companies", Journal of Small Business Finance, vol. 3, no. 2, pp.111-125.

24. Meggison, W.L., Nash, R.C., Netter, J.M., and Poulsen, A.B. (2004), "The Choice of Private Versus Public Capital Markets: Evidence from Privatizations", The Journal of Finance, vol. 59, no. 6, pp 2835-2870.

25. Nikoskelainen, E., and Wright, M. (2007), "The impact of corporate governance mecha- 
nisms on value increase in leveraged buyouts", Journal of Corporate Finance, vol. 13, no. 4, pp. 511-537.

26. Schmidt, D. (2006), "Private equity versus stocks: Do the alternative asset's risk and return characteristics add value to the portfolio?", Journal of Alternative Investments, vol. 9, no. 1, pp. 28-47.

27. Schmidt, D., Nowak, E., and Knigge, A. "On the Performance of Private Equity Investments: Does Market Timing Matter?” Working Paper, 2004.

28. Phalippou, L., and Gottschalg, O. (2009), “The performance of private equity funds" Review of Financial Studies, vol. 22, no. 4, pp. 1747-1776.

29. Phalippou, L., and Zollo, M. (2005), "What Drives Private Equity Fund Performance?", SSRN working paper. 